\title{
Method Construction by Goal Analysis
}

\author{
C. Gonzalez-Perez ${ }^{1}$, P. Giorgini ${ }^{2}$ and B. Henderson-Sellers ${ }^{3}$ \\ 1 University of Technology, Sydney, Department of Software Engineering, cesar- \\ gon@verdewek.com \\ 2 University of Trento, Department Information and communication Technology, \\ paolo.giorgini@unitn.it \\ 3 University of Technology, Sydney, Department of Software Engineering, \\ brian@it.uts.edu.au
}

\begin{abstract}
Method engineering proposes the construction of methodologies by selecting method fragments from a repository and assembling then in an appropriate way. However, the rules by which the "optimal" method fragments are chosen are not clear, and such chores are usually done manually by an expert. This paper presents a goal analysis technique for the selection of the optimal method fragments from a repository, using backward reasoning to obtain the set of fragments that satisfy the desired goals with minimum effort. By using this technique, a methodologist can determine the goals that the organisation wants the methodology to satisfy, and then, preferably, rely on automated tools for the selection of the optimal solution.
\end{abstract}

\section{Introduction}

It is well accepted that no single software development methodology (or method; we will consider them here as synonyms) serves all purposes (Cockburn 2000). Different project, product and organisational characteristics call for different methodologies, which are often further tweaked or customised to fit the particular idiosyncrasies of its users (Bajec, Vavpotič, and Krisper 2007). One quick way to obtain a customised methodology is to adopt an existing one and change it as necessary. However, this entails significant risks since the methodologists making the changes are not necessarily aware of the interconnections and dependencies between different components of the methodology. The situational method engineering (SME) paradigm (Brinkkemper 1996; Henderson-Sellers, Serour, McBride, Gonzalez-Perez, and Dagher 2004b) offers a solution to this problem: instead of adopting an existing methodology and changing it as necessary, a custom methodology is created by selecting the appropriate method fragments from an existing repository and combining them appropriately. This approach is used in methodological frameworks such as OPF (Firesmith and Henderson-Sellers 2002), OOSPICE (Henderson-Sellers, Stallinger, and Lefever 2002) and FIPA (Cossentino, Gaglio, Garro, and Seidita 2007), 
and is advocated in the recent ISO/IEC (2007) 24744 International Standard "Software Engineering Metamodel for Development Methodologies".

Despite an increasing and broadening interest in situational method engineering, some areas are still to be fully explored. For example, how are the method fragments to be selected from the repository? A complete methodology is likely to be composed of hundreds of method fragments, and each of these must be carefully chosen to (a) fit the purpose of the methodology being constructed and (b) be compatible with other method fragments. Usually, this task is performed by a methodologist, who uses his/her expert judgement to handcraft an "optimal" solution. This approach has a number of drawbacks. First of all, it can be extremely time consuming. Secondly, there is no way to demonstrate that the chosen collection of method fragments is best, i.e. no guarantee can be given on the quality of the result (other than that given by the trust on the methodologist's expertise). Typically, an organisation willing to adopt the method engineering paradigm will need to recruit a methodologist or hire a consultant to compose a methodology each time.

This paper presents a solution to these drawbacks in which a project manager will be able to create a profile of the methodology to be constructed in terms of the goals that it must achieve, and then use a goal analysis technique, ideally implemented by a tool, to extract the optimal combination of method fragments from the repository that fulfils the goals at minimum effort.

In the rest of this paper, Section 2 introduces some important concepts of method engineering; Section 3 explains the basic concepts of goal analysis and Section 4 its application to methodology construction.

\section{Background for Situational Method Engineering}

As explained above, the SME approach needs the existence of a method fragment repository. This repository is usually a database that contains method fragments of different kinds. Method fragments are self-contained, relatively independent specifications of some aspect of a methodology, such as a task to be performed, a technique that may be employed, a product that can be generated or a team that can be formed. Different kinds of method fragments have different properties: for example, task specifications have a purpose (that declares what the task intends to achieve) and a description (that specifies the steps that may be followed in order to achieve it); work product specifications, on the other hand, have a name (such as "Requirements Specification Document" or "Class Diagram") and a description. In turn, different kinds of method fragments are related to each other: for example, task specifications may be linked to the work products that they generate when executed.

The structure of the repository, i.e. the kinds of method fragments, their properties and the relationships between them, is usually given by a metamodel. A metamodel is a formal description of the concepts that can be used to construct a methodology and the relationships amongst them. Here, we will adopt the International Standard ISO/IEC (2007) 24744 "Software Engineering Metamodel for Develop- 
ment Methodologies" (SEMDM). SEMDM defines 68 concrete classes, instances of which can potentially be stored in a method fragment repository. Not all the method fragment classes are relevant for this paper; we will concentrate on the following:

- PhaseKind. Specification of a managed timeframe within a project for which the objective is the transition between levels of abstraction. Phase kinds specify the "when" of a methodology, i.e. its temporal ordering and organisation.

- ProcessKind. Specification of a discrete, large-grained job performed within a project that operates within a given area of expertise. Process kinds specify the "what and why" of a methodology at an abstract level, i.e. the methodology's job structure.

- TaskKind. Specification of a small-grained job performed within a project that focuses on what must be done in order to achieve a given purpose. Task kinds specify the "what and why" of a methodology at a detailed level.

- TechniqueKind. Specification of a small-grained job performed within a project that focuses on how the given purpose may be achieved. Technique kinds specify the "how" of a methodology, i.e. the specific means of achieving the associated task.

- WorkProductKind. Specification of an artefact of interest for the project. Work product kinds specify what is created and consumed during a project.

- ActionKind. Specification of how a given task kind acts upon a particular work product kind.

These classes are interrelated in the following way (Fig. 1) i.e. each phase kind is composed of process kinds, which give "content" to it. The phase kind specifies when something must be done, while the associated process kinds define what to do. In turn, each process kind contains a number of task kinds, which flesh out and refine the process' purpose. In turn, each task kind may be associated to a number of technique kinds, since there is often a choice from several techniques, each of which can be used to achieve the goals of the same task, and different tasks can use the same technique. Finally, each task kind may be mapped to a number of work product kinds via action kinds. These mappings involve different action types: a task can create, modify, delete or read a work product. Typically, each task kind will read work products of some kinds and perhaps create a new work product of a different kind.

\subsection{Sample Method Fragment Repository}

Consider the following (simplified) example. Two phase kinds are defined in a repository: "System Definition" and "System Construction". The first is intended to be performed at the beginning of a project and defines the system to be built. The second is meant to be executed at the end of a project in order to construct the system previously defined. A number of process kinds are also defined: "Requirements Engineering", "Coding", "Acceptance Testing", "Quality Assurance" and "Process Improvement". Each of these process kinds specifies, from an abstract point of view, what can be done at some point in the project. Some of these process kinds are associated to the "System Definition" phase kind, some to "System Construction", and 
some to both (Table 1). Then, some task kinds can be introduced, such as "Elicit requirements", "Analyse requirements", "Validate requirements", "Develop service models" and "Determine work product defects" (Table 2). These task kinds, together with many more, would be associated to different process kinds. A number of technique kinds can also be introduced, such as "Prototyping", "Peer reviewing" and "Threat modelling" (Table 3). These technique kinds would be mapped to task kinds in a many-to-many fashion. Finally, some work product kinds can be introduced into the repository, such as "Requirements Specification Document", "Service Diagram", "Source Program" and "Report" (Table 4). Each of these work product kinds would be associated to a number of task kinds with a particular action type; for example "Requirements Specification Document" can be mapped to "Document requirements" via an action kind with a "create" type and to "Develop service models" via a different action kind with a "read" type. In turn, "Service Diagram" can be mapped to "Develop service models" via an action kind with a "create" type.

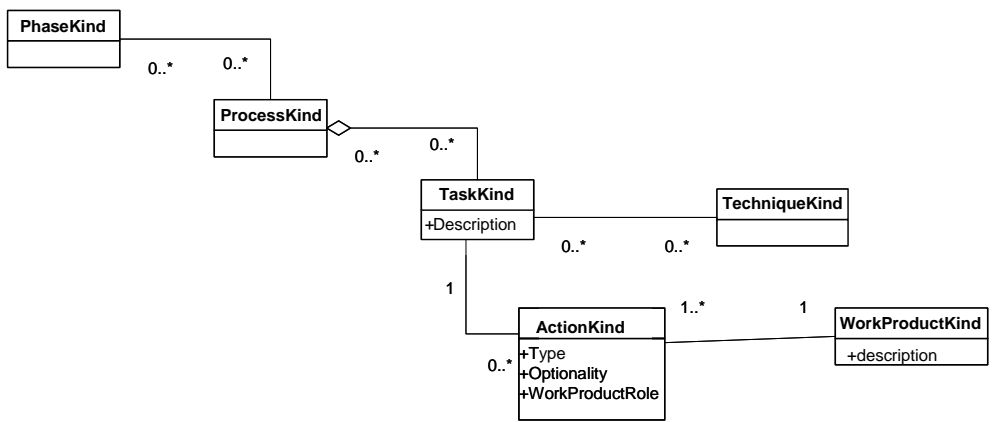

Fig. 1. Metamodel fragment (a subset of ISO/IEC 24744). Only relevant classes, attributes and associations are depicted. Here the diamond indicates a generic whole-part relationship.

Relevant life cycle models are created by instantiating the class TimeCycleKind from ISO/IEC 24744. This is a subtype of StageWithDurationKind (also the supertype of PhaseKind). Selection of the lifecycle is a stylistic decision much akin to the choice of architectural style for an software application. While it is possible that we can represent this selection process in terms of a soft goal, it is more likely that the choice will be made based on other, external factors and influences. (This topic of life cycle selection is a topic for future research - not discussed further here.)

Table 1 Sample process kinds.

\begin{tabular}{ll}
\hline Name & Mapped to phase kinds \\
\hline Requirements Engineering & System Definition \\
\hline Coding & System Construction \\
\hline Acceptance Testing & System Construction \\
\hline Quality Assurance & System Definition, System Construction \\
\hline Process Improvement & System Definition, System Construction \\
\hline
\end{tabular}


Table 2 Sample task kinds.

\begin{tabular}{ll}
\hline Name & Mapped to process kinds \\
\hline Elicit requirements & Requirements Engineering \\
\hline Analyse requirements & Requirements Engineering \\
\hline Validate requirements & Requirements Engineering \\
\hline Document requirements & Requirements Engineering \\
\hline Develop class models & High-Level Modelling \\
\hline Develop service models & High-Level Modelling \\
\hline Sketch user interface & High-Level Modelling \\
\hline Develop interaction models & Detailed Modelling \\
\hline Write code & Coding \\
\hline Unit test class & Coding \\
\hline Demonstrate the system & Acceptance Testing \\
\hline Obtain stakeholder feedback & Acceptance Testing, Quality Assurance \\
\hline Determine work product defects & Quality Assurance \\
\hline Prepare defect report & Quality Assurance \\
\hline Test build system & Quality Assurance \\
\hline
\end{tabular}

Table 3 Sample technique kinds.

\begin{tabular}{ll}
\hline Name & Mapped to task kinds \\
\hline Prototyping & Develop service models, Sketch user interface \\
\hline Text analysis & Analyse requirements, Develop class models \\
\hline CRC cards & Develop class models \\
\hline Peer reviewing & Validate requirements, Determine work product defects \\
\hline Test-first development & Unit test class \\
\hline In-house customer & Demonstrate the system, Obtain stakeholder feedback \\
\hline Automated builds & Test build system \\
\hline Threat modelling & Analyse requirements \\
\hline
\end{tabular}

Table 4 Sample work product kinds with action types.

\begin{tabular}{lll}
\hline Name & Mapped to task kinds & Action type \\
\hline Stakeholders & Elicit requirements & create \\
\cline { 2 - 3 } Statement & Analyse requirements, Validate requirements & modify \\
\cline { 2 - 3 } & Document requirements & read \\
\hline Requirements & Document requirements & create \\
\cline { 2 - 3 } $\begin{array}{l}\text { Specification } \\
\text { Document }\end{array}$ & Develop class models, Develop service models, & read \\
\hline Service Diagram & Sketch user interface & \\
\cline { 2 - 3 } & Develop service models & create \\
\cline { 2 - 3 } & Develop interaction models & modify \\
\hline User Interface & Sketch user interface & read \\
\cline { 2 - 3 } Sketch & Develop service models & create \\
\cline { 2 - 3 } & Write code & modify \\
\hline Source Program & Write code & create \\
\cline { 2 - 3 } & Unit test class & modify \\
\cline { 2 - 3 } & Test build system & read \\
\hline Report & Test build system, Determine work product de- & create \\
& fects, Prepare defect report & \\
\hline
\end{tabular}


From the sample method fragments in Tables 1-4, it can be seen that the dependency network that can arise from the method fragments in a repository can be extremely intricate. For example, selecting the "High-Level Modelling" process kind would usually imply bringing along the "Develop service models" task kind, which "creates" a "Service Diagram" work product and "reads" a "Requirements Specification Document" work product. In order to provide the necessary input (i.e. a requirements specification document), we need to select a task kind that creates it, namely "Document Requirements". This task kind, in turn, may bring along the whole "Requirements Engineering" process kind together with additional task kinds.

Technique selection is usually more flexible, since a number of technique kinds are often available for each individual task kind. Which is selected depends only on the characteristics of the project (e.g. time or budget constraints), the product context (e.g. safe-criticality) and the organisation (e.g. culture and skills). Although we can assume that any of the technique kinds mapped to a given task kind is appropriate to achieve the task's purpose, the particular technique kinds that are chosen will likely influence overall project properties such as time consumed or defect injection rate as well as providing a different level of risk and associated costs. From this perspective, we can say that some techniques are better than others for some particular purposes.

\subsection{Requirements for Method Construction}

The design and construction of a methodology can be seen as any other engineering activity: some requirements are given and a suitable artefact that satisfies them must be developed. Therefore, we can assume that some requirements exist when methodologists face the task of constructing a methodology from a method fragment repository. These requirements can be described in terms of the capabilities and qualities of the intended outcome of the engineering effort, namely, the future methodology. In turn, method capabilities and qualities may refer to the kind of products that the method can construct, the type of projects used to tackle such activities and the characteristics of the organisations where these projects may take place. If we can characterise products, projects and organisations with measurable attributes, we will have a solid starting point on which requirements for method construction can be defined. These can be seen as defining the requirements for the construction of the methodology (as opposed to the requirements for the construction of the software application, which is the target of the software development project) (Ralyté 2002). Factors that influence these requirements are many, including organizational maturity level, skills set of development team members, type of domain (e.g. information systems, realtime control, e-business), project size, team size, level of criticality, interface style, level of resources allocated to project and whether or not the system is to be a distributed application (Nguyen and Henderson-Sellers 2003)

Table 5 shows a list of the attributes that we have identified for the purpose of illustration in this paper. We have only included attributes that may be directly affected by the choice of method fragments when constructing a methodology. We are aware that many other attributes (such as product correctness or readability) are also 
of interest to software engineering, but they have been left out from this experiment since they are not likely to be directly affected by the choice of method fragments.

Table 5 Product, project and organisation attributes for method construction.

\begin{tabular}{|c|c|c|}
\hline Area & Attribute & Description \\
\hline \multirow[t]{3}{*}{ Product } & Reliability & $\begin{array}{l}\text { The product must offer high reliability, i.e. its users } \\
\text { will depend on it for critical operations. }\end{array}$ \\
\hline & Changeability & $\begin{array}{l}\text { The product will need to be changed, so it will need } \\
\text { to offer the appropriate mechanisms to achieve this } \\
\text { with ease. }\end{array}$ \\
\hline & Usability & The product must be easy to use. \\
\hline \multirow[t]{4}{*}{ Project } & Cost constraints & $\begin{array}{l}\text { The project has cost constraints, so it must be } \\
\text { completed at the lowest cost possible. }\end{array}$ \\
\hline & Time constraints & $\begin{array}{l}\text { The project has time constraints, so it must be } \\
\text { completed in the shortest time possible. }\end{array}$ \\
\hline & Staffing constraints & $\begin{array}{l}\text { The project has staffing constraints, so it must be } \\
\text { completed with the lowest possible number of staff. }\end{array}$ \\
\hline & Visibility & $\begin{array}{l}\text { The project needs high visibility, so all the work } \\
\text { must be properly documented. }\end{array}$ \\
\hline \multirow[t]{3}{*}{ Organisation } & Formal culture & $\begin{array}{l}\text { The development team's culture promotes formal, } \\
\text { high-ceremony work. }\end{array}$ \\
\hline & Agile culture & $\begin{array}{l}\text { The development team's culture promotes agile- } \\
\text { style, low-ceremony work. }\end{array}$ \\
\hline & Experience & $\begin{array}{l}\text { The development team has got extensive experience } \\
\text { in the kind of project and product to be developed. }\end{array}$ \\
\hline
\end{tabular}

\section{Goal Analysis Concepts}

In goal analysis, the final goal of each process step is considered from the point of view of a specific actor. There are three relevant reasoning techniques that are useful: means-end analysis, contributions analysis and AND/OR decomposition (Bresciani, Giorgini, Giunchiglia, and Mylopolous 2004). In means-end analysis, the following are performed iteratively until an acceptable solution is reached: "Describe the current state, the desired state (the goal) and the difference between the two; Select a promising procedure for enabling this change of state by using this identified difference between present and desired states; Apply the selected procedure and update the current state." (Henderson-Sellers, Giorgini, and Bresciani 2004a)

Contributions analysis helps to identify goals that may contribute towards the partial fulfilment of the final goal and is sometimes used as an alternative to meansend analysis, particularly useful for softgoals. Positive or negative influences towards attainment of the goal are identified and quantified on a (usually 5 point) Likert scale. In particular, contribution analysis has been shown to be very effective for soft goals used for eliciting non-functional (quality) requirements. 
Finally, AND/OR decomposition changes a root goal into a finer goal structure i.e. a set of subgoals - either alternatives (OR decomposition) or additive (AND decomposition).

Goal analysis has been used in a number of ways to support software development e.g. in the design of systems, especially for documenting early requirements, as in the Tropos methodology (Bresciani et al., 2004); in business process reengineering (Grau, Franch, and Maiden 2005); and in the support of ISO/IEC15504 assessments (Rifaut, 2005). Here, we present the first application of goal analysis to method construction in the context of method engineering.

\section{Applying Goal Analysis to Method Construction}

In order to use goal analysis for method construction, we need to determine how each of the method fragments in the sample repository affects each of the above listed attributes. For example, we can say that performing the Quality Assurance process (see Table 1) enhances product reliability. For each method fragment plus attribute pair, one of five possible values has been determined: strongly enhances, enhances, neutral, deteriorates and strongly deteriorates.

Table 6 shows these (non-neutral) mappings between method fragments and attributes. Please note that we are not claiming that these mappings are optimal or even correct; these are a sample collection of reasonable mappings for the purpose of this paper. A separate study would be necessary in order to determine how each method fragment in a production repository affects each attribute of interest.

Suppose we have two options for a Software Engineering Process (SEP) and each has several Tasks, each implemented by a Technique chosen from a list. The two options are shown graphically in Figure 2.

Table 6 Mappings between attributes and method fragments. For each mapping, a value is included indicating how the choice of the method fragment affects the attribute.

\begin{tabular}{|c|c|c|c|c|}
\hline \multirow{2}{*}{$\begin{array}{l}\text { Attribute } \\
\text { Area }\end{array}$} & \multicolumn{3}{|c|}{ Method Fragment } & \multirow[t]{2}{*}{ Value } \\
\hline & Name & Class & Name & \\
\hline \multirow[t]{8}{*}{ Product } & \multirow[t]{5}{*}{ Reliability } & Process kind & Quality Assurance & $\begin{array}{l}\text { strongly } \\
\text { enhances }\end{array}$ \\
\hline & & Task kind & Unit test class & enhances \\
\hline & & \multirow[t]{3}{*}{ Technique kind } & Test-first development & enhances \\
\hline & & & In-house customer & enhances \\
\hline & & & Threat modelling & $\begin{array}{l}\text { strongly } \\
\text { enhances }\end{array}$ \\
\hline & \multirow[t]{2}{*}{ Changeability } & Process kind & $\begin{array}{l}\text { Configuration Man- } \\
\text { agement }\end{array}$ & enhances \\
\hline & & Task kind & $\begin{array}{l}\text { Document require- } \\
\text { ments }\end{array}$ & enhances \\
\hline & Usability & Process kind & Acceptance Testing & $\begin{array}{l}\text { strongly } \\
\text { enhances }\end{array}$ \\
\hline
\end{tabular}




\begin{tabular}{|c|c|c|c|c|}
\hline \multirow[t]{3}{*}{ Attribute } & & \multicolumn{2}{|l|}{ Method Fragment } & \multirow{2}{*}{$\begin{array}{l}\text { Value } \\
\text { enhances }\end{array}$} \\
\hline & & Task kind & $\begin{array}{l}\text { Demonstrate the } \\
\text { system }\end{array}$ & \\
\hline & & & $\begin{array}{l}\text { Obtain stakeholder } \\
\text { feedback }\end{array}$ & $\begin{array}{l}\text { strongly } \\
\text { enhances }\end{array}$ \\
\hline \multirow[t]{13}{*}{ Project } & \multirow{3}{*}{$\begin{array}{l}\text { Cost con- } \\
\text { straints }\end{array}$} & Phase kind & System Definition & deteriorates \\
\hline & & Process kind & Quality Assurance & deteriorates \\
\hline & & & Process Improvement & deteriorates \\
\hline & \multirow{5}{*}{$\begin{array}{l}\text { Time con- } \\
\text { straints }\end{array}$} & Phase kind & System Definition & deteriorates \\
\hline & & Process kind & Process Improvement & deteriorates \\
\hline & & Task kind & Unit test class & deteriorates \\
\hline & & Technique kind & Prototyping & deteriorates \\
\hline & & & Automated builds & enhances \\
\hline & \multirow{3}{*}{$\begin{array}{l}\text { Staffing con- } \\
\text { straints }\end{array}$} & Process kind & Quality Assurance & deteriorates \\
\hline & & Technique kind & Peer reviewing & deteriorates \\
\hline & & & Pair programming & deteriorates \\
\hline & \multirow[t]{2}{*}{ Visibility } & \multirow[t]{2}{*}{ Task kind } & Prepare defect report & enhances \\
\hline & & & $\begin{array}{l}\text { Prepare process qual- } \\
\text { ity report }\end{array}$ & enhances \\
\hline \multirow[t]{15}{*}{ Organisation } & \multirow[t]{2}{*}{ Formal culture } & Phase kind & System Definition & $\begin{array}{l}\text { strongly } \\
\text { enhances }\end{array}$ \\
\hline & & Task kind & $\begin{array}{l}\text { Measure process } \\
\text { quality }\end{array}$ & enhances \\
\hline & \multirow[t]{5}{*}{ Agile culture } & Phase kind & System Definition & $\begin{array}{l}\text { strongly } \\
\text { deteriorates }\end{array}$ \\
\hline & & Process kind & Process Improvement & deteriorates \\
\hline & & Task kind & $\begin{array}{l}\text { Document require- } \\
\text { ments } \\
\text { Elicit requirements }\end{array}$ & $\begin{array}{l}\text { deteriorates } \\
\text { enhances }\end{array}$ \\
\hline & & \multirow[t]{2}{*}{ Technique kind } & In-house customer & enhances \\
\hline & & & Test-first development & enhances \\
\hline & \multirow[t]{8}{*}{ Experience } & Phase kind & System Definition & $\begin{array}{l}\text { strongly } \\
\text { enhances }\end{array}$ \\
\hline & & \multirow[t]{2}{*}{ Process kind } & $\begin{array}{l}\text { Requirements Engi- } \\
\text { neering }\end{array}$ & enhances \\
\hline & & & Acceptance Testing & enhances \\
\hline & & Task kind & Elicit requirements & enhances \\
\hline & & \multirow[t]{4}{*}{ Technique kind } & Focus groups & $\begin{array}{l}\text { strongly } \\
\text { enhances }\end{array}$ \\
\hline & & & Prototyping & $\begin{array}{l}\text { strongly } \\
\text { enhances }\end{array}$ \\
\hline & & & Walkthroughs & enhances \\
\hline & & & In-house customer & enhances \\
\hline
\end{tabular}

Looking at the Techniques we have a table (akin to Table 6 above) that links Techniques to impact factors (-ilities). The Techniques are labelled as X1-X6 where $\mathrm{X} 1$ = Test first; X2 = In house customer; X3 = Prototyping; X4 = Automated builds; $\mathrm{X} 5=$ Threat modelling; and $\mathrm{X} 6=$ Peer reviewing. Then the two processes can be described in terms of these terminal Techniques as: 
$\mathrm{SEP} 1$ is $(\mathrm{X} 1$ or $\mathrm{X} 2)$; $(\mathrm{X} 3$ or $\mathrm{X} 4)$

$\mathrm{SEP} 2$ is $(\mathrm{X} 1$ or $\mathrm{X} 2)$; ( $\mathrm{X} 1$ or $\mathrm{X} 2$ or $\mathrm{X} 5$ or $\mathrm{X} 6)$

We consider just two examples. The impact on the Reliability and of Agility factors:

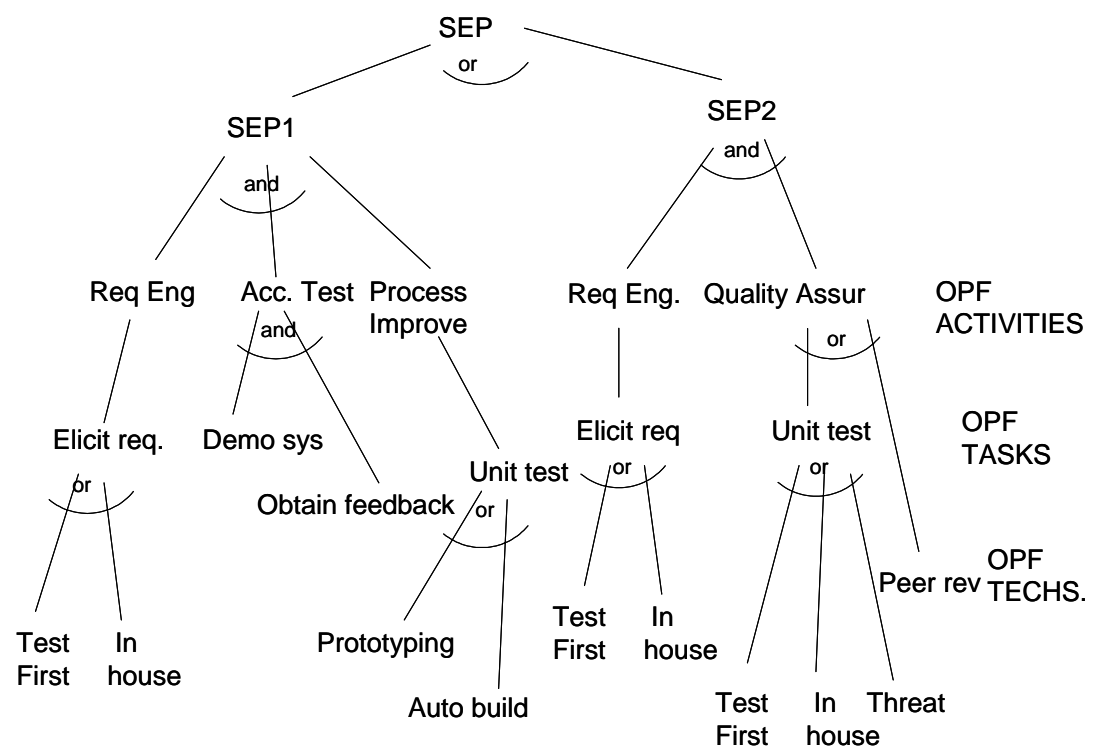

Fig. 2 Hierarchical tree depicting Activities, Tasks and Techniques for two hypothetical SEPs

1) reliability

Test-first development (X1)

enhances $(+)$

In-house customer (X2)

Prototyping (X3)

Automated builds (X4)

Threat modelling(X5)

Peer reviewing (X6)

2) agility

Test-first development (X1)

In-house customer (X2)

Prototyping (X3)

Automated builds (X4)

Threat modelling(X5)

Peer reviewing (X6

enhances $(+)$

neutral (o)

deteriorates (-)

strongly enhances $(++)$

strongly enhances $(++)$

enhances $(+)$

enhances $(+)$

deteriorates (-)

strongly deteriorates (--)

strongly deteriorates (--)

strongly enhances $(++)$

Then the impact is as follows:

OPTION

SEP1 option 1 is $\mathrm{X} 1$; $\mathrm{X} 3$

Reliability

$+/ 0$

Agility

$+1-$ 


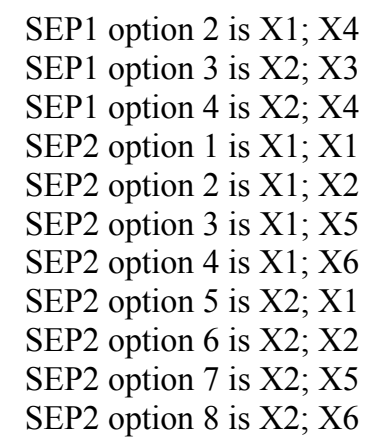

$\begin{array}{ll}+/- & +/-- \\ +/ 0 & +/- \\ +/- & +/-- \\ +/+ & +/+ \\ +/+ & +/+ \\ +/++ & +/- \\ +/++ & +/++ \\ +/+ & +/+ \\ +/+ & +/+ \\ +/++ & +/-- \\ +/++ & +/++\end{array}$

We conclude that from a reliability viewpoint, the best choice would be SEP2, options $3,4,7$ or 8 . On the other hand, from an agility perspective, the best choice would be SEP2, option 4 or 6 .

The above analysis is fully supported and automated in Tropos (Giorgini, Mylopoulous, and Sebastiani 2005). In particular, backward reasoning allows the analyst to search for possible method fragments from the repository that satisfy the desired goal. Moreover, by assigning a cost to each fragment, backward reasoning also produces the solution with the minimum cost.

\section{Conclusions and Future Work}

With the aim of creating a high quality software development methodology from those method fragments selected from an existing repository, we have examined a new idea based on goal analysis. Rather than select the elements of the methodology "top-down" by considering what seems reasonable in a particular situation using what might be termed "intuition" (the current approach in SME), we suggest that a more objective process can be created in which the main focus becomes the goal rather than the means of achieving that goal (the process element). The goal analysis approach proposed here permits the creation of an optimized methodology; importantly, one that is optimized for a particular characteristic such as reliability or agility. An hierarchical tree is constructed (Figure 2) and, for each element, we identify whether there is a positive or negative impact for the chosen optimization characteristic. We have demonstrated this approach with a simple example of a small tree in which fragments for activities, tasks and techniques from the OPF repository have been selected, considering the impacts on two different software engineering processes, SEP1 and SEP2 (Figure 2). That these processes have different optima under different evaluation criteria (here agility and reliability) suggests that this approach is worthy of further investigation including practical trials in industry and the development of a prototype support tool. We are currently planning such industry trials within the Italian ministry funded project MEnSA (http://www.mensa-project.org) project and anticipate building appropriate support tools in due course. 


\section{Acknowledgments}

We wish to thank for financial support both the Australian Research Council and the Italian ministry for research through its PRIN-MEnSA project.

\section{References}

Bajec, M., Vavpotič, D. and Krisper, M. (2007) Practice-driven approach for creating projectspecific software development methods. Inf. Software Technol. 49, 345-365.

Bresciani, P., Perini, A., Giorgini, P., Giunchiglia, F. and Mylopolous, J. (2004) Tropos: an agent-oriented software development methodology. Autonomous Agents and Multi-Agent Systems 8(3), 203-236

Brinkkemper, S. (1996) Method engineering: engineering of information systems development methods and tools. Inf. Software Technol. 38(4), 275-280.

Cockburn, A.S. (2000) Selecting a project's methodology. IEEE Software 17(4), 64-71.

Cossentino, M., Gaglio, S., Garro, A. and Seidita, V. (2007) Method fragments for agent design methodologies: from standardization to research. Int. J. Agent-Oriented Software Eng. 1(1), 91-121

Firesmith, D.G. and Henderson-Sellers, B. (2002) The OPEN Process Framework. AddisonWesley, London.

Giorgini P., Mylopoulous J. and Sebastiani R. (2005). Goal-oriented requirements analysis and reasoning in the Tropos methodology. Eng. Appl. Artific. Intell. 18(2), 159-171.

Grau, G., Franch, X. and Maiden, N.A.M. (2005) A goal-based round-trip method for system development. In: Procs. 11th International Conference on Requirements Engineering: Foundations for Software Quality (REFSQ'05), pp. 67-82.

Henderson-Sellers, B., Stallinger, F. and Lefever, B. (2002) Bridging the gap from process modelling to process assessment: the OOSPICE process specification for componentbased software engineering. In: Procs. $28^{\text {th }}$ EUROMICRO Conference. Dortmund, Germany, 4-6 September 2002. IEEE Computer Society: Los Alamos, CA, USA, pp. 324-331.

Henderson-Sellers, B., Giorgini, P. and Bresciani, P. (2004a) Enhancing Agent OPEN with concepts used in the Tropos methodology. In: A. Omicini, P. Pettra and J. Pitt (Eds.), Engineering Societies in the Agents World IV. 4th International Workshop, ESAW 2003. LNAI 3071, Springer-Verlag, Berlin, pp. 328-345.

Henderson-Sellers, B., Serour, M. McBride, T. Gonzalez-Perez, C. and Dagher, L. (2004b) Process construction and customization. J. Universal Computer Science. 10(4), 326-358.

ISO/IEC (2007). Software Engineering Metamodel for Development Methodologies. ISO/IEC 24744, International Organization for Standardization, Geneva.

Nguyen, V.P. and Henderson-Sellers, B. (2003) Towards automated support for method engineering with the OPEN Process Framework. In: M.H Hamza (Ed.), Procs. Seventh IASTED International Conference on Software Engineering and Applications. ACTA Press, Anaheim, CA, USA, pp. 691-696.

Ralyté, J. (2002) Requirements definition for the situational method engineering. In: C. Rolland, S. Brinkkemper and M. Saeki (Eds.), Engineering Information Systems in the Internet Context. Kluwer Academic Publishers, Boston, USA, pp. 127-152.

Rifaut, A. (2005) Goal-driven requirements engineering for supporting the ISO 15504 assessment process. In: I. Richardson, P. Abrahamsson and R. Messnarz (Eds.), Software Process Improvement. $12^{\text {th }}$ European Conf., EuroSPI 2005, LNCS 3792, Springer, pp. 151-162 